\title{
Langmuir Films of Anthracene Derivatives on Liquid Mercury I: Symmetric Molecules
}

\author{
L. Tamam \\ Department of Physics, Bar-Ilan University, Ramat-Gan 52900, Israel
}

H. Kraack

Department of Physics, Bar-Ilan University, Ramat-Gan 52900, Israel

E. Sloutskin

Department of Physics, Bar-Ilan University, Ramat-Gan 52900, Israel

\section{B. M. Ocko}

Department of Physics, Brookhaven National Laboratory, Upton, New York 11973

\author{
P. S. Pershan \\ Department of Physics and DEAS, Harvard University, Cambridge, Massachusetts 02138 \\ E. Ofer \\ Department of Physics, Bar-Ilan University, Ramat-Gan 52900, Israel
}

\author{
M. Deutsch* \\ Department of Physics, Bar-Ilan University, Ramat-Gan 52900, Israel
}

Received: June 23, 2006; In Final Form: November 28, 2006

\begin{abstract}
The structure and phase sequence of liquid-mercury-supported Langmuir films (LFs) of two symmetric acenes, anthracene and anthraquinone, were studied by surface tensiometry and X-ray diffraction. At low coverage, both form a monolayer of surface-parallel, flat-lying, molecules. At high coverage, we find a monolayer of side-lying molecules, where the molecular plane is surface-normal, and the molecular long axis is surfaceparallel. None of these phases exhibit long-range in-plane order.
\end{abstract}

\section{Introduction}

For the last century, monolayers of organic films on aqueous and solid surfaces have been studied widely. This interest is driven by their technological applications and scientific importance. ${ }^{1}$ These systems allow one to study molecular interactions, structural organization, rheology, transport phenomena, and so forth in quasi-two-dimensional matter. The advent of synchrotron sources and liquid surface spectrometers over the last two decades allowed for the development of surface-specific X-ray scattering techniques for studying the structure of liquid surfaces in general $^{2,3}$ and, in particular, of these films in situ, under a variety of conditions, with angstrom-scale resolution. ${ }^{4}$

The first modern-era studies of water-supported monolayers were published in the late 19th century. ${ }^{5}$ In such films, which were later named "Langmuir films" (LFs), the molecules must be amphiphilic. The intermolecular van der Waals interactions, along with the partial hydrophobicity, always align the organic molecules roughly normal to the surface, with the hydrophilic headgroup residing on the water and the hydrophobic tails pointing away from it. The order within the film can be tuned

\footnotetext{
* Corresponding author. E-mail: deutsch@mail.biu.ac.il.
}

by varying the surface coverage, the temperature, the molecule's conformation, structure and dimensions, and by additives to the subphase.

Another class of monolayers is self-assembled mono- and multilayers on solid substrates (SAMs), mostly thiols on gold and silanes on $\mathrm{Si} / \mathrm{SiO}_{2}$ surfaces. ${ }^{6,7}$ Unlike those on water, on these solid substrates phases with surface-parallel molecular orientations were found. SAMs also differ from LFs in the headgroup-subphase bonding. These energies in SAMs (a few hundred kilojoules per mole) are substantially higher than the energy of the hydrogen bonds of the head groups to the water subphase in LFs (a few kilojoules per mole). The different nature of the substrates of LFs and SAMs, and the difference in the strength of the bonding affect differently the surface-parallel structure within the films. The mobile, short-range-ordered molecules of the water substrate, which virtually lacks a corrugation potential, do not impose an order on LFs, and their in-plane order is dominated, therefore, by the intermolecular interactions within the LF. In contrast, in SAMs, the in-plane order is dominated by the epitaxy to the crystalline substrate's intrinsic order through its corrugation potential. Thus, the resultant order of a SAM may differ from that of a LF even when the molecule and surface coverage are the same. 
The liquid-mercury-supported LFs studied here, and in several previous $^{8-13}$ studies, are an intermediate case between LFs on aqueous subphases and SAMs on a crystalline metal substrate. The Hg subphase's atomically smooth surface, short-range liquid order, atomic mobility, and lack of corrugation potential are very similar to those of water. At the same time, the types of interactions of organic molecules with a mercury subphase, and their strengths, closely resemble those of the SAMs' molecules with their solid substrate.

Polycyclic aromatic hydrocarbons, like the acenes studied here, have been increasingly investigated over the past decade as macroscopic organic replacements for the inorganic semiconductor materials ( $\mathrm{Si}, \mathrm{Ge}, \mathrm{GaAs}$, etc.) employed today by the electronics industry. ${ }^{14}$ The budding research field of molecular electronics is also greatly interested in such molecules for nanosize and single-molecule electronic devices. ${ }^{15}$ The favorable electronic properties of these molecules stem from their relatively high number of nonlocalized electrons in the $\pi$ bonds of the conjugated rings. The overwhelming majority of the structural studies to date of monolayers of such molecules have been carried out on solid substrates, which, as discussed above, do not always reflect the intrinsic structure favored by the intermolecular interactions within the SAM. Schreiber ${ }^{16}$ and Witte and Wöll ${ }^{17}$ review recent work in the field, ranging over films of perylenes, phtalocyanines, coronenes, polythiophenes, and polyacenes on a range of semiconductor ( $\mathrm{Si}, \mathrm{GaAs}$ ), insulator $\left(\mathrm{SiO}_{2}, \mathrm{Al}_{2} \mathrm{O}_{3}, \mathrm{MgO}\right.$, alkalihalides), and metal ( $\mathrm{Au}-$ (111), $\mathrm{Au}(110), \operatorname{Ag}(111), \operatorname{Ag}(110), \mathrm{Cu}(110))$ substrates. Rubrene was also studied recently on both $\mathrm{SiO}_{2}$ and $\mathrm{Au}(111) .{ }^{18}$ Of the polyacenes, pentacene has been studied most because of its ability to form (by evaporation and by organic molecular-beam deposition) well-ordered films with high charge mobilities and other favorable transport properties. ${ }^{19}$

These studies concluded that the structure is dominated by the balance between two major interactions. A flat-lying pentacene molecule maximizes the contact of its $\pi$ orbitals with the electronic orbitals (or band structure) of the substrate. However, surface-normal orientation of the molecular plane maximizes the overlap of the $\pi$ orbitals of adjacent pentacene molecules. ${ }^{20,21}$ Thus, on weakly interacting substrates like $\mathrm{SiO}_{2},{ }^{19,21}$ or alkanethiol-derivative-coated metallic surfaces, ${ }^{20}$ the $\pi-\pi$ overlap of adjacent molecules dominates, orienting the pentacene molecules normal to the surface, and stacking them face-to-face. In contrast, on strongly interacting substrates, like the metals $\mathrm{Au}, \mathrm{Ag}$, and $\mathrm{Cu}$, the interaction of the penatcene's $\pi$ orbital with the substrate dominates, and the pentacene molecules lie flat on the surface. ${ }^{22-24}$ In multilayers on strongly interacting metal surfaces, the first layer is flat-lying, while subsequent layers, having progressively weaker interaction with the surface, are driven by the strong $\pi-\pi$ interactions to a bulklike packing, where the molecular plane of the pentacene is no longer surface-parallel. ${ }^{25}$ Finally, we note that on crystalline substrates the surface-parallel structure of the pentacene monolayer is determined by epitaxy to the crystalline substrate's structure, even when this means considerably reduced packing as compared to that of the bulk crystalline phase of pentacene. ${ }^{20,23,26}$ In some cases, multilayers assembled on such polyacene monolayers exhibit novel 3D structures. ${ }^{26,27}$

These results demonstrate the dominant role of the substrate's properties in the determination of the polyacene monolayer's structure. As we show, this interaction plays a major role in the present study: the liquid mercury substrate's lack of longrange order and corrugation potential, and its strong interaction with the thiol headgroup result in the emergence of novel (a)

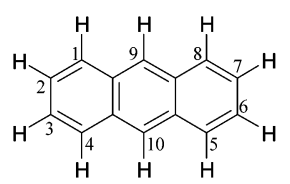

(b)

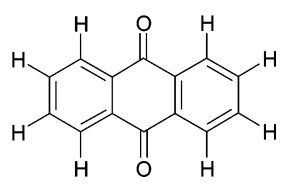

Figure 1. Molecular structure of the molecules studied. (a) Anthracene, showing the numbering convention of the carbons; (b) anthraquinone.

structures, not observed on solid substrates, which can be varied in situ by increasing the surface coverage.

The only previous angstrom-resolution measurement of the structure of an aromatic monolayer on a liquid-metal subphase is our recent study of LFs of biphenyl thiols on mercury. ${ }^{13}$ At low coverage a condensed, but in-plane disordered, single layer of surface-parallel molecules is found for the two compounds studied. At high coverage, in-plane-ordered phases of standingup molecules were found. As expected, the structures found on liquid mercury differ from those on solid Au in several important respects. Twisting of the two phenyl rings relative to each other around their single $\mathrm{C}-\mathrm{C}$ bond is an important motif in the structure of biphenyl monolayers. This rotational flexibility is not available for the acene molecules studied here, where adjacent rings share two carbons and thus also a common molecular plane. As we show below, this leads to different structural features in the two systems.

A more comprehensive approach to the self-assembly of molecular films, in the absence of epitaxy, involves a balance between several interactions: molecule-molecule interaction, head group-head group interaction, molecule-subphase interaction, and head group-subphase interaction. Changes in one or more of these interactions may lead to a different structure of the monolayer. Our previous studies of films of alkane derivatives on mercury ${ }^{8,9,11}$ demonstrate that a change of the headgroup leads to different molecular packings, phase diagrams, and transition temperatures and pressures. The different anthracene derivatives studied here were chosen with the aim of investigating the role of functional groups, and their positions within the molecule, on the assembly process of the monolayer and the resultant structure of the monolayer.

The four different anthracene derivatives chosen for the present study can be divided into two groups according to the Lewis structure of the molecule. The first group comprises anthracene and anthraquinone, shown in Figure 1, which have mirror symmetry with respect to both the central long and short axes of the molecule. Anthracene, $\mathrm{C}_{14} \mathrm{H}_{10}$, consists of three connected benzene rings. The dimensions of the bounding box of the van der Waals surface of the anthracene molecule have been calculated to be $11.65 \times 7.44 \times 3.88 \AA^{3}{ }^{328}$ This is the smallest bounding box of the four molecules addressed in this study because the three other anthracene derivatives include additional moieties that increase one or more of their dimensions. Anthraquinone, $\mathrm{C}_{14} \mathrm{H}_{8} \mathrm{O}_{2}$, contains, in addition, two opposite carbonyl groups $(\mathrm{C}=\mathrm{O})$ at carbon nos. 9 and 10 . The molecule is close to planar with the $\mathrm{C}=\mathrm{O}$ bond inclined at an angle of $3.8^{\circ}$ to the plane of the rings. ${ }^{29}$

The second group, anthrone and anthralin, lack mirror symmetry with respect to the long axis of the molecules, although the mirror symmetry with respect to the central short axis is preserved. These are discussed in an accompanying paper. ${ }^{30}$ The LFs of the two groups show, on mercury, fundamentally different structural features, highlighting the importance of the molecular conformation on that of the monolayer.

The study presented here employs surface tensiometry to determine the pressure-area isotherms of LFs. Surface-specific 
X-ray diffraction techniques were employed to determine the surface-normal and surface-parallel structure of the LFs as a function of surface coverage. The experimental methods used are described in the next section, followed by a section presenting the results obtained for the symmetric molecules. The last section of this paper provides a short summary. The results for the two asymmetric molecules are presented in the following paper, ${ }^{30}$ where the overall implications of the results obtained for all molecules are discussed in detail.

\section{Experiment}

The experimental setup, and the measurement and data analysis methods, have been described previously in detail. ${ }^{8-11,13}$ Thus, only a brief description will be given here. The surface thermodynamics were explored using surface pressure $\pi-$ molecular area $A$ isotherms. The films' structure was studied by synchrotron-based surface X-ray reflectivity and grazingincidence diffraction. Both types of measurements employ a diffractometer-mountable Langmuir trough, which allows one to carry out simultaneous surface tension and X-ray measurements.

A. Instrumentation and Materials. $\mathrm{Hg}$ was purchased from Merck Co. (triple distilled, 99.999\%) and Bethlehem Apparatus Co. (quadruple distilled, 99.9995\%). The materials studied, anthracene and its derivatives, were purchased from Aldrich and Alfa-Aeser (purity: $\geq 97 \%$ ) and used as received.

Spreading solutions, of $3-8 \times 10^{-4}$ molarity, were prepared using HPLC grade, $99.9 \%$ pure, chloroform (Aldrich). Chloroform was found in previous experiments to evaporate rapidly, leave no traces of its own, and yield reproducible isotherms. ${ }^{8,10,12,31}$

The trough was machined from KelF and has an area of 6.5 $\times 17.5 \mathrm{~cm}^{2}$. It is enclosed in a sealed box, having Kapton windows for entry and exit of X-rays. The box was filled with helium (X-ray measurements) or nitrogen (isotherm measurements) to reduce surface contamination and beam damage. For $\mathrm{X}$-ray measurements, the box was attached to an active vibration isolation unit, mounted on the diffractomemter. This practically eliminated vibrational pickup from the environment. $8,10,12,31$

The Wilhelmy plate method ${ }^{32}$ was used to measure the surface tension, employing a $\mathrm{Hg}$-amalgamated platinum plate. The homemade balance employed a leaf spring, the changes in the position of which, due to surface tension changes, were measured by a linear variable differential transformer (LVDT). A well-sealing barrier is notoriously difficult to construct for mercury. ${ }^{33}$ Thus, the area per molecule, $A$, was varied not by barrier compression but rather by a stepwise addition, by a calibrated micropipette, of microliter volumes of the spreading solution. After one portion was deposited, we waited until a full pressure equilibration was reached before deposition the next portion. ${ }^{9}$ The trough's temperature was controlled to $\pm 0.2{ }^{\circ} \mathrm{C}$ by a commercial water circulator.

B. Measurement Methods. 1. Isotherms. The surface pressure, $\pi=\sigma_{0}-\sigma$, is the difference between the surface tension of the uncovered, $\sigma_{0}$, and film-covered, $\sigma$, mercury. It depends on the surface coverage, $A$, measured as the area per molecule. The isotherm $\pi(A)$ provides information on the phases and phase transitions of the Langmuir film.

2. X-ray Diffraction. The structure of the LF was studied by surface-specific X-ray techniques, employing the Harvard/BNL liquid surface diffractometer, Beamline X22B, National Synchrotron Light Source, Brookhaven National Laboratory, at a wavelength $\lambda=1.539 \AA$. For details of the setup and procedures, see refs $34-37$

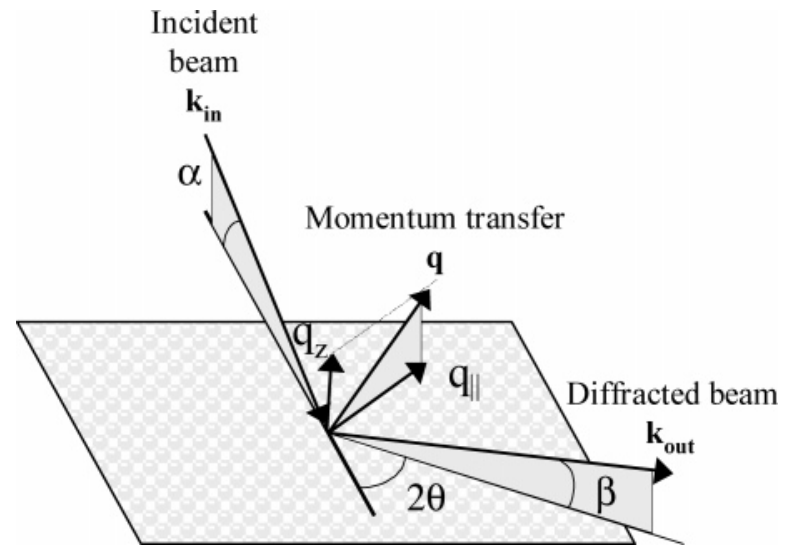

Figure 2. X-ray scattering geometry.

The X-ray diffraction geometry is shown in Figure 2. Three types of measurement were carried out: X-ray reflectivity (XR), where the fraction of the incident intensity reflected specularly, $R\left(q_{z}\right)$, is measured as a function of the surface-normal wave vector transfer, $q_{z}=(2 \pi / \lambda)(\sin \alpha+\sin \beta) . \alpha$ and $\beta$ are the incidence and detection angles, respectively, and $\alpha=\beta$. XR yields information on the surface-normal structure of the LF such as the surface-normal electron density profile, the layer's thickness, and the surface roughness. The in-plane order is studied by grazing incidence diffraction (GID). Here, the incidence angle is kept constant at a value $\alpha \sim 0.8 \alpha_{c}$, where $\alpha_{c}$ is the critical angle for total external reflection. Our $\alpha_{c} \approx$ $0.47^{\circ}$ corresponds to a critical momentum transfer of $q_{\mathrm{c}} \approx(4 \pi)$ ג) $\alpha_{c}=0.067 \AA^{-1}$. The diffracted intensity is measured as a function of the surface-parallel wave vector transfer $q_{\|}=(2 \pi /$ d) $\sqrt{\cos ^{2} \alpha+\cos ^{2} \beta-2 \cos \alpha \cos \beta \cos 2 \theta}$. Here, $2 \theta$ is the surfaceparallel angular offset of the detector from the reflection plane. If surface-parallel order exists within the LF, diffraction peaks will be observed at angles $2 \theta$ that correspond through the Bragg law to the repeat distances of the structure. The peak positions allow one to determine the unit cell and surface-parallel structure of the film, just like in conventional X-ray diffraction. The distributions of the intensities along $q_{z}$ at the $q_{\|}$positions of the GID peaks, denoted as Bragg rods (BRs), provide information on the magnitude and azimuthal direction of the molecular tilts. The BR is characterized by a sharp surface-enhancement peak at $q_{z}=q_{\mathrm{c}}$ (the Vineyard peak ${ }^{38}$ ) on top of a broader peak. This broad peak's width is inversely related to the length of the molecule, while its peak position is related to the tilt angle of the molecule. ${ }^{36}$

XR measurements employ a NaI scintillation point detector while the GID measurements use a surface-normal-aligned position sensitive linear detector. This detector allows for a simultaneous measurement of a full BR at the $2 \theta$ position of each GID peak. An upstream automated shutter, opened only during the actual counting time at each measurement point, was used to minimize beam damage to the LF.

C. Modeling of the X-ray Results. The XR curves are analyzed using fits by a previously employed box model, describing the surface-normal electron density. ${ }^{8,9,11,12}$ One or two "boxes" represent the organic overlayers (a single or double layer in this study), and seven boxes represent the layered mercury subphase. ${ }^{39}$ Each box has a width, a constant height (electron density), and a roughness at its interfaces with the adjacent boxes.

The electron density of an overlayer box can be calculated by dividing the number of electrons in the molecule by the molecular volume. For the ordered monolayer phases, the 


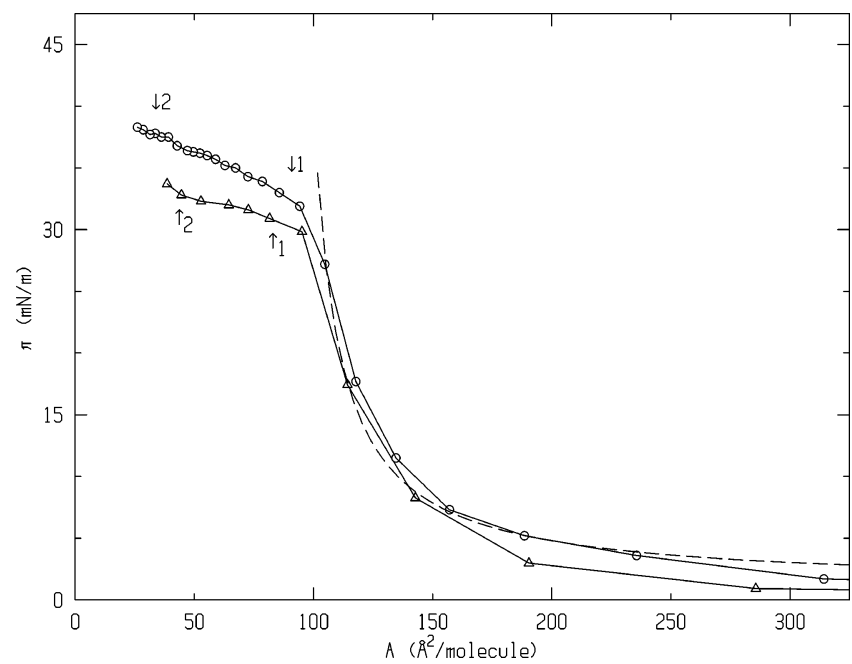

Figure 3. Measured $\pi-A$ isotherms of LFs of anthracene (circles) and anthraquinone (triangles) on mercury. A fit of the anthracene isotherm by the Vollmer equation, the isotherm of a 2D hard-disk gas, is shown in a dashed line. Arrows mark the coverages where X-ray measurements were done for each compound.

molecular volume is obtained accurately from the unit cell dimensions derived from the GID pattern. For the disordered phase, where no GID peaks are observed, the volume can be calculated from the dimensions of the molecules, and slight variations in this value were allowed in the fit to achieve good fits. The width of the box (representing the thickness of the film) was varied in the fit, either as a free fit parameter, or, when strongly correlated with other fit parameters, as a fixed, but manually varied, value in an iterative series of fits.

The widths of the seven boxes representing the layered density profile of the mercury subphase were fixed at $1.3 \AA$, half the atomic layer spacing at the mercury surface. ${ }^{10,31}$ All interfacial roughness values between these boxes were fixed at $0.7 \AA$. The electron densities of the boxes were varied, except for the first box, which was fixed at $(5.5 \pm 0.2) \mathrm{e} / \AA^{3}$, an average of many trial fits for different reflectivity curves. The mercury's bulk electron density (the last box) was also fixed at the $3.25 \mathrm{e} / \AA^{3}$, calculated from the room-temperature mass density of mercury. Our previous studies of LFs on mercury $8,9,11-13$ demonstrated that these procedures yield very good fits, and consistent, physically reasonable results. As discussed below, and shown in Figure 4, this model provides excellent fits to the present data as well, over a broad $q$-range and 8-9 orders of magnitude in the reflectivity, $R\left(q_{z}\right)$.

For those LFs that exhibit GID peaks, each peak is fitted by a single Lorentzian, from which the peak position, widths, and integrated intensity could be calculated. The peak positions are used to obtain the order and unit cell dimensions, while the peak width provides information on the crystalline coherence length of the ordered domains. The BR curves were fitted by a previously used model assuming a uniform electron density distribution along the molecular long axis..$^{35,36}$

\section{Results}

We discuss here the results obtained for anthracene and anthraquinone. These molecules have mirror symmetry with respect to both the molecular long axis and the molecular short axis, which runs normal to the long axis in the plane of the molecule. The structure of these molecules is shown in Figure 1.

A. Isotherms. The room-temperature $\left(T=24{ }^{\circ} \mathrm{C}\right)$ measured pressure-area $(\pi-A)$ isotherms of anthracene and anthraquinone
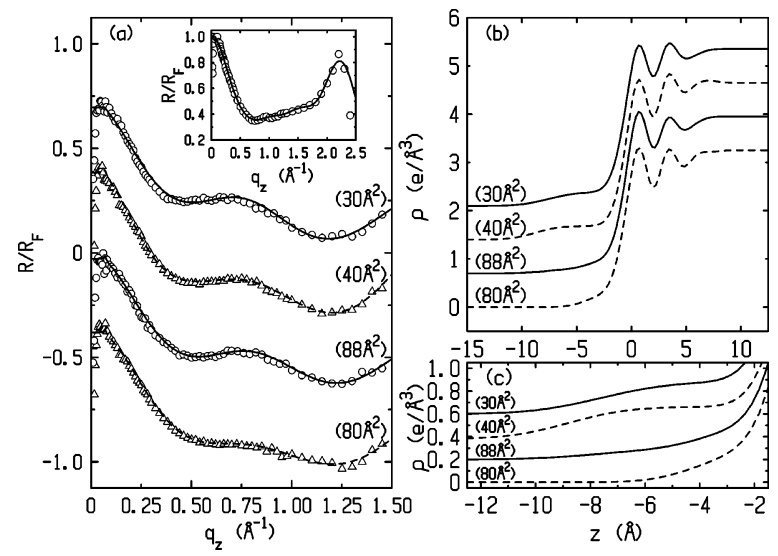

Figure 4. (a) Measured Fresnel-normalized X-ray reflectivity curves of LFs of anthracene (circles) and anthraquinone (triangles) on mercury at the indicated nominal areas per molecule. The corresponding box model fits are also shown (lines). Curves are upshifted by 0.4 each for clarity. Inset: the measured reflectivity (circles) and the box model fit (line) for the bare $\mathrm{Hg}$ surface. (b) The electron density profiles obtained from the fits in part a. Anthracene is shown by solid lines and the anthraquinone is shown by dashed lines. Curves are upshifted by 0.7 each for clarity. (c) The monolayer part of $b$ on an enlarged scale. Curves are upshifted by 0.2 each for clarity.

on mercury are shown in Figure 3. The curves are very similar and differ only in the height of their plateaus at $A \leq 100 \AA^{2 /}$ molecule.

A reduction of $A$ from $300 \AA^{2} /$ molecule to $150 \AA^{2} /$ molecule produces only a very small change in the surface pressure, as expected for a dilute 2D gas of molecules. However, below 150 $\AA^{2} /$ molecule a very steep rise in the surface pressure is observed, from $\pi \approx 8 \mathrm{mN} / \mathrm{m}$ at $150 \AA^{2} / \mathrm{molecule}$ to $\pi \approx 35 \mathrm{mN} / \mathrm{m}$ at 100 $\AA^{2} /$ molecule. The larger- $A$ part of the isotherm, $100 \leq A \leq$ $500 \AA^{2} /$ molecule, was fitted by the Vollmer equation, $\pi(A-$ $\left.A_{0}\right)=k_{\mathrm{B}} T$, where $k_{\mathrm{B}}$ is the Boltzmann constant and $A_{0}$ is the exclusion area due to the finite size of the molecule. ${ }^{10}$ Good fits result, as shown for anthracene in the dashed line in Figure 3 . These fits yield exclusion areas of $A_{0}=(90 \pm 3) \AA^{2} /$ molecule for anthracene and $A_{0}=(92 \pm 2) \AA^{2} /$ molecule for anthraquinone. The $A_{0}$ of anthracene is very close to the calculated area of the anthracene molecules in the bulk ${ }^{28} A_{\text {calc }}^{\mathrm{lw}}=l \times w \approx$ $12 \times 7.5=90 \AA^{2}$, where $l$ and $w$ are the molecular length and width, respectively. This hints that the molecules are oriented parallel, or almost parallel, to the surface for $A \gtrsim 90 \AA^{2} /$ molecule, and the fast rise in $\pi$ at $90 \AA^{2} /$ molecule is associated with the formation of a densely packed single layer (SL) phase of flat-lying molecules. This conclusion is supported by the $\mathrm{X}$-ray results discussed below. The slightly larger exclusion area of the anthraquinone could arise from the oxygen side groups, which may require more space than the hydrogens of the anthracene. An oxygen-moiety-induced increase in the effective thickness of anthrone and anthralin, akin to the width increase of the anthraquinone postulated here, was found in the GID measurements reported in ref 30 . Upon decreasing $A$ below 90 $\AA^{2} /$ molecule, a (slow-rising) plateau is observed in the isotherms. Such plateaus are usually associated with a coexistence region between two phases. ${ }^{34}$

The isotherm of anthraquinone is qualitatively very similar to that of anthracene, with the onset of the "plateau" appearing at roughly the same molecular area $A$. The plateaus have similar slopes, with that of the anthraquinone being somewhat lower. The film collapse seems to occur for anthracene at a somewhat lower $A$ than that for anthraquinone, although no definite statement to that effect can be made. These similarities between 
the isotherms indicate a similar structure for the LFs of anthracene and anthraquinone. This is borne out by the X-ray results, discussed in the next sections.

B. Surface-Normal Structure. Figure 4a shows typical XR curves (open symbols) measured at $24{ }^{\circ} \mathrm{C}$ and normalized by the Fresnel reflectivity $R_{F}\left(q_{z}\right)$ of an ideally flat and abrupt surface. ${ }^{36}$ The inset of Figure 4 a shows the same curve for the bare mercury surface. The peak at $q_{z} \approx 2.2 \AA^{-1}$ is due to the surface-induced layering of the mercury. ${ }^{39}$ To explore both the low- and high-coverage regions of the isotherms, the XR measurements for the LFs of anthracene and anthraquinone were carried out at the onset of the "plateau", at $A=88$ and $80 \AA^{2} /$ molecule, respectively, and at slightly less than half these areas. The lines in Figure 4a show the model fits for anthracene (solid line) and anthraquinone (dashed line), with the corresponding electron density profiles shown in the same lines in Figure 4b. For anthraquinone, XR measurements were also carried out at $4.7{ }^{\circ} \mathrm{C}$. The $\mathrm{XR}$ curves were nearly identical with those measured at room temperature. In addition, no significant differences were observed between XR curves measured $\sim 0.5-1 \mathrm{~h}$ after film deposition and those measured up to $6.5 \mathrm{~h}$ after deposition at the same temperature and coverage.

The period, $\Delta q_{z}$, of the modulations in $R / R_{F}$ is inversely related to the LF's thickness: $d=2 \pi / \Delta q_{z}$, and should allow, in principle, to derive a rough estimate for the layer thickness from the ruler-measured period, even without a detailed modeling. However, this calculation is valid only when several periods of equal $\Delta q_{z}$ are observed within the measurement-accessible $q_{z}$ range. In the present case, the layer thickness is small, and, thus, the modulation period is large. Consequently, the rise in the reflectivity for $q_{z} \gtrsim 1.5 \AA^{-1}$, due to the mercury layering, pushes the position of the second minimum toward lower $q_{z}$ values. This results in a shorter apparent modulation period, and a larger apparent layer thickness, than those of the layer. This effect stands out when comparing the minima positions in the upper two, low- $A$, curves, with those of the lower two, high$A$, curves in Figure 4a. The first minimum is observed to shift down considerably in $q_{z}$ upon the reduction of $A$. Because the thickness of the lower- $A$ film is larger than that of the higher- $A$ one, it is expected that as $A$ decreases the second minimum will shift down in $q_{z}$ even more, to make the period smaller. This, however, does not occur, and the second minimum stays at roughly the same position, regardless of the decrease in $A$. This results in the anomalous effect that if the observed minima are used to determine $\Delta q_{z}$ and $d$ then a layer thickness larger by $\sim 20 \%$ is obtained at higher- $A$ than at lower- $A$. The model fits, however, take into account correctly the effect of the mercury layering on the reflectivity curve, and provide, therefore, more reliable thickness values for the layer. Even so, the $\mathrm{XR}$ curve distortion due to the layering peak results in the inducement of some correlations between the fit-refined parameters, if all are free to vary. This, in turn, results, for example, in a non-negligible uncertainty in the value of $d$, particularly for the thinner layers at low coverage. Considering that the exclusion areas agree so well with the calculated area for a molecule oriented with its benzene rings parallel to the surface, we carried out fits of the low coverage XR curves assuming this orientation, and keeping $d$ fixed at the known $4 \AA$ thickness of the molecule. These are the fits shown in lines for $A=88$ $\AA^{2} /$ molecule (anthracene) and $A=80 \AA^{2} /$ molecule (anthraquinone) in Figure $4 \mathrm{a}$. As can be observed, the fits obtained are good. The resultant electron densities, $\rho=(0.28 \pm 0.02) \mathrm{e} / \AA^{3}$ for anthracene and $\rho=(0.29 \pm 0.02) \mathrm{e} / \AA^{3}$ for anthraquinone, agree well with those calculated from the molecular dimensions.
Finally, the roughness values, discussed below, are close to those of pure mercury.

We conclude, therefore, that at the onset of the plateau both the measured isotherm and the XR results are consistent with the LF being a single layer of close-packed molecules, oriented with the molecular plane parallel to the surface, or very nearly so. This conclusion holds for both anthracene and anthraquinone.

The high-coverage XR curves in Figure 4 are similar, though not identical, to those measured at low coverage, for reasons discussed above. This requires careful consideration of the model and the procedures employed in their fits. Thus, extensive series of fits, with different combinations of fixed and fitted parameters, were carried out in an attempt to obtain a reliable layer thickness and density values. First, a single uniform-density organic layer was assumed to reside on top of the mercury subphase. The parameters defining the mercury subphase were kept fixed at the values refined in the low-coverage fits. This did not allow us to obtain a good fit to the measured XR of either the anthracene or the anthraquinone for any combination of the other parameters, the layer thickness, density, and top and bottom roughnesses. However, allowing a variation of two of the seven layers defining the $\mathrm{Hg}$ surface (layers 2 and 4 from the top) resulted in the good fits shown in Figure 4, with only small variations in the $\mathrm{Hg}$ density profile. For all fits of the anthracene XR curves for which the variation of the layered $\mathrm{Hg}$ profile from that of the low-coverage fits was small, the thickness of the high-coverage anthracene layer converged to $\sim 7.6 \AA$, and the density converged to $\sim 0.28 \mathrm{e} / \AA^{3}$. Attempts to keep the layer thickness fixed at the low coverage value of $d=$ $4 \AA$ failed to produce a good fit to the measured XR curve. The density obtained agrees well with that calculated from the molecular volume and the number of electrons in the molecule. The value of $d$ is close to the width of the anthracene molecule, implying that the anthracene molecule is lying on its side: its long axis is surface-parallel, and the molecular plane is roughly normal to the surface. The thickness $d$ of the layer, obtained from the fit, could also correspond to the formation of a double layer of flat-lying molecules. Thus, we employed a second model, where the high-coverage layer was modeled by two layers, the lower one of a fixed density of $0.28 \mathrm{e} / \AA^{3}$ and a thickness of $4 \AA$ (i.e., a $100 \%$ coverage by lying-down molecules) and the top one of a varying density. A fit of the anthracene XR at $A=30 \AA^{2} /$ molecule yielded a density of $(0.28$ $\pm 0.01) \mathrm{e} / \AA^{3}$ and thickness of $(3.8 \pm 0.1) \AA$ for the top layer. Distinguishing this model of two $d \approx 4 \AA$ equal-density layers from a $d \approx 8 \AA$ single layer of the same density is, of course, impossible. However, previous studies of LF on mercury of other molecules exhibiting such two-layer phases ${ }^{9,11}$ invariably show in the isotherm an additional jump to a second, higher, plateau when such a double-layer phase (DL) of flat-lying molecules occurs. This can be observed, for example, in Figure 2 in ref 9. Such features are not observed in the present isotherm, Figure 3. We conclude, therefore, that it is unlikely that the high-coverage phase of anthracene consists of a double layer of flat-lying molecules.

A similar analysis to that described above was applied to the nominal $A=40 \AA^{2} /$ molecule XR curve of anthraquinone. The difference between the high- and low-coverage XR curves for anthraquinone is more pronounced than that for anthracene, indicating a greater change in the fitted profile, as can indeed be observed in Figure 4c. The fit yields a layer thickness $d=$ $(8.8 \pm 0.1) \AA$ and an electron density of $\rho=(0.28 \pm 0.01)$ $\mathrm{e} / \AA^{3}$. The molecular dimensions of the anthraquinone are the same as those of the anthracene, except for the width, which is 
larger by $\sim 1.3 \AA$, corresponding to the carbon-oxygen bond length of the side group. ${ }^{40}$ Thus, the XR fits imply a side-lying orientation for anthraquinone, same as that argued above for anthracene. The electron density obtained from the fit is in good agreement with that obtained from the calculated molecular volume and the 102 electrons of the molecule: $\rho_{\text {calcd }} \approx 102 /$ $(12 \times 3.8 \times 8.8)=0.27 \mathrm{e} / \AA^{3}$. The possibility that the $d=8.8$ $\AA$ thickness of the anthraquinone film is due to a double layer of flat-lying molecules is, again, unlikely, for the same reasons detailed above for anthracene.

Finally, we note that the fits yield surface roughness values in the range of $(1.6 \pm 0.4) \AA$, only slightly higher than the capillary-wave-induced $1.4 \AA$ roughness of the pure mercury. ${ }^{31}$

C. Surface-Parallel Structure. The anthracene GID was measured only at room temperature, $T=24{ }^{\circ} \mathrm{C}$. The measurements for anthracene were carried out for one sample at three coverages and repeated several times up to $3 \mathrm{~h}$ after deposition. For anthraquinone, they were carried out on two samples, several times per sample in the time interval between 1.5 and $6.5 \mathrm{~h}$ after deposition, at two different coverages for each of the two temperatures measured, $T=24{ }^{\circ} \mathrm{C}$ and $T=4.7{ }^{\circ} \mathrm{C}$. The $q_{\|}$ range scanned was $0.2 \leq q_{\|} \leq 1.7 \AA^{-1}$. No GID peaks were observed in any of these measurements. This indicates that the surface-parallel order in these LFs is short-ranged and extends over a few molecular diameters only.

\section{Discussion}

Mercury-supported LFs of both anthracene and anthraquinone exhibit the same structure: flat-lying molecules for molecular areas down to $A \approx 90 \AA^{2} /$ molecule, and side-lying molecules for $A<90 \AA^{2} /$ molecule. Because no GID peaks are found, it is concluded that no in-plane long-range order exists in these LFs.

The collapse pressure of a LF of the 14-carbon anthracene, $\sim 40 \mathrm{mN} / \mathrm{m}$, is $30 \%$ higher than that of a LF of the 14-carbon alkane, tetradecane, which is only $\leq 30 \mathrm{mN} / \mathrm{m}$. ${ }^{9}$ This can be rationalized by noting that the ring structure of the anthracene molecule results, when side-lying, in the formation of an effective double layer of carbons. This double layer produces, per unit area, a net van der Waals attraction to the mercury surface that is higher than that of the single-layer of carbons formed by packing extended, flat-lying alkane molecules on the surface. An exact numerical comparison will require taking into account the distance dependence of the attractive potential in the surface-normal direction and the distances from the surface of all carbons of an anthracene molecule. Although the latter is easy to calculate, the former is not known with a sufficient level of accuracy. The strong overlap of the $\pi$ orbitals of adjacent molecules provided by the surface-normal orientation of the molecular planes may also contribute to the increased resistance to, and higher pressure required for, film collapse.

The oxygen moieties of the anthraquinone appear to have no influence on the LF structure, as compared to that of an anthracene LF, other than increasing the exclusion area slightly, and the layer thickness in the side-lying phase. Also, no net dipole moment is expect to be induced by the oxygens because of their symmetric positions, on carbon nos. 9 and 10 . In contrast, a rather large dipole moment is induced in the anthrone and anthralin molecules, where the oxygens are attached to one side of the molecule only. The consequences of these difference are discussed in the next paper. ${ }^{30}$

Acknowledgment. Support to M.D. by the U.S.-Israel Binational Science Foundation, Jerusalem and to P.S.P. by the NSF (grant No. DMR-0124936) is gratefully acknowledged. We thank D. Vaknin (Ames) for generous advice and drawings of the trough. BNL is supported by U.S. DOE Contract No. DEAC02-98CH10886.

\section{References and Notes}

(1) (a) Ulman, A. An Introduction to Ultrathin Organic Films; Academic: Boston, MA, 1991. (b) Schreiber, F. Prog. Surf. Sci. 2000, 65, 151. (c) Ulman, A. Thin Films: Self-Assembled Monolayers of Thiols; Academic: San Diego, CA, 1998.

(2) (a) Als-Nielsen, J.; Christensen, F.; Pershan, P. S. Phys. Rev. Lett. 1982, 48, 1107. (b) Pershan, P. S.; Als-Nielsen, J. Phys. Rev. Lett. 1984, 52, 759. (c) Als-Nielsen, J.; Jacquemain, D.; Kjaer, K.; Leveiller, F.; Lahav, M.; Leiserowitz, L. Phys. Rep. 1994, 246, 252. (d) Penfold, J. Rep. Prog. Phys. 2001, 64, 777.

(3) Braslau, A.; Deutsch, M.; Pershan, P. S.; Weiss, A. H.; Als-Nielsen, J.; Bohr, J. Phys. Rev. Lett. 1985, 54, 114.

(4) The first three in situ X-ray studies of LMs were published almost simultaneously: (a) Wolf, S. G.; Leiserowitz, L.; Lahav, M.; Deutsch, M.; Kjaer, K.; Als-Nielsen, J. Nature 1987, 328, 63. (b) Kjaer, K.; Als-Nielsen, J.; Helm, C. A.; Laxhuber, L. A.; Mohwald, H. Phys. Rev. Lett. 1987, 58 2224. (c) Dutta, P.; Peng, J. B.; Lin, B.; Ketterson, J. B.; Prakash, M.; Georgopoulos, P.; Ehrlich, S. Phys. Rev. Lett. 1987, 58, 2228.

(5) Pockels, A. Nature 1891, 43, 437.

(6) Leung, T. Y. B.; Schwartz, P.; Scoles, G.; Schreiber, F.; Ulman, A. Surf. Sci. 2000, 458,34 .

(7) Sagiv, J. J. Am. Chem. Soc. 1980, 102, 92.

(8) (a) Kraack, H.; Ocko, B. M.; Pershan, P. S.; Deutsch, M. Science 2002, 298, 1404. (b) Kraack, H.; Deutsch, M.; Ocko, B. M.; Pershan, P. S. Nucl. Instrum. Methods Phys. Res., Sect. B 2003, 200, 363.

(9) Kraack, H.; Ocko, B. M.; Pershan, P. S.; Sloutskin, E.; Deutsch M. J. Chem. Phys. 2003, 119, 10339.

(10) Kraack, H.; Ocko, B. M.; Pershan, P. S.; Sloutskin, E.; Tamam, L.; Deutsch M. Langmuir 2004, 20, 5375.

(11) Kraack, H.; Ocko, B. M.; Pershan, P. S.; Sloutskin, E.; Tamam, L.; Deutsch M. Langmuir 2004, 20, 5386.

(12) Ocko, B. M.; Kraack, H.; Pershan, P. S.; Sloutskin, E.; Tamam, L.; Deutsch M. Phys. Rev. Lett. 2004, 94, 017802.

(13) Tamam, L.; Kraack, H.; Sloutskin, E.; Ulman, A.; Pershan, P. S.; Deutsch, M.; Ocko, B. M. J. Phys. Chem. B 2005, 109, 12534.

(14) (a) Yang, Y.; Heeger, A. J. Nature 1994, 372, 344. (b) Facchetti, A.; Mushrush, M.; Yoon, M-H.; Hutchison, G. R.; Ratner, M. A.; Marks, T. J. J. Am. Chem. Soc. 2004, 126, 13859. (c) Crone, B.; Dodabalapur, A.; Lin, Y. Y.; Filas, R. W.; Bao, Z.; LaDuca, A.; Sarpeshkar, R.; Katz, H. E.; Li, W. Nature 2000, 403, 521. (d) Dodabalpur, A.; Torsi, L.; Katz, H. E. Science 1995, 268, 270. (e) Dimitrakopoulos, C. D.; Mascaro, D. J. IBM J. Res. Dev. 2001, 45, 11.

(15) (a) Mantooth, B. A.; Weiss, P. S. Proc. IEEE 2003, 91, 1785. (b) Molecular Electronics; Jortner, J., Ratner, M., Eds.; Blackwell: Oxford, 1997.

(16) Schreiber, F. Phys. Status Solidi A 2004, 201, 1037.

(17) Witte, G.; Wöll, Ch. J. Mater. Res. 2004, 19, 1889.

(18) Käfer, D.; Ruppel, L.; Witte, G.; Wöll, Ch. Phys. Rev. Lett. 2005, 95,166602 .

(19) (a) Ruiz, R.; Choudhary, D.; Nickel, B.; Toccoli, T.; Chang, K.C.; Mayer, A. C.; Clancy, P.; Blakely, J. M.; Headrick, R. L.; Iannotta, S.; Malliaras, G. G. Chem. Mater. 2004, 16, 4497. (b) Ruiz, R.; Mayer, A. C.; Malliaras, G. G.; Nickel, B.; Scoles, G.; Kazimirov, A.; Kim, H.; Headrick, R. L.; Islam, Z. Appl. Phys. Lett. 2004, 85, 4926.

(20) (a) Hu, W. S.; Tao, Y. T.; Hsu, Y. J.; Wei, D. H.; Wu, Y. S. Langmuir 2005, 21, 2260. (b) Yamamoto, T. Synlett 2003, 4, 425.

(21) Fritz, S. E.; Martin, S. M.; Frisbie, C. D.; Ward, M. D.; Toney, M. F. J. Am. Chem. Soc. 2004, 126, 4048.

(22) Eremtchenko, M.; Temirov, R.; Bauer, D.; Schaefer, J. A.; Tautz, F. S. Phys. Rev. B 2005, 72, 115430 .

(23) Floreano, M.; Cossaro, A. S.; Cvetko, D.; Bavdek, G.; Morgante, A. J. Phys. Chem. B 2006, 110, 4908.

(24) Lukas, S.; Witte, G.; Wöll, Ch. Phys. Rev. Lett. 2002, 88, 028301. (25) (a) Witte, G.; Wöll, Ch. Phase Transitions 2003, 76, 291. (b) Chen, Q.; McDowell, A. J.; Richardson, N. V. Langmuir 2003, 19, 10164.

(26) (a) Wang, Y. L.; Ji, W.; Shi, D. X.; Du, S. X.; Seidel, C.; Ma, Y. G.; Gao, H.-J.; Chi, L. F.; Fuchs, H. Phys. Rev. B 2004, 69, 075408. (b) Lu, B.; Zhang, H. J.; Huang, H.; Mao, H. Y.; Chen, Q.; Li, H. Y.; He, P.; Bao, S. N. Appl. Surf. Sci. 2005, 245, 208.

(27) Kang, J. H.; Zhu, X. Y. Appl. Phys. Lett. 2003, 82, 3248.

(28) Sander, L. C.; Wise, A. Polycyclic Aromatic Hydrocarbon Structure Index; NIST Special Publication 922; NIST: Gaithersburg, MD, 1997.

(29) Murthy, B. V. R. Acta Crystallogr. 1957, 10, 146.

(30) Tamam, L.; Kraack, H.; Sloutskin, E.; Ocko, B. M.; Pershan, P. S.; Ofer, E.; Deutsch, M. J. Phys. Chem. C 2007, 111, 2580.

(31) DiMasi, E.; Tostmann, H.; Ocko, B. M.; Pershan, P. S.; Deutsch, M. Phys. Rev. B 1998, 58, R13419. 
(32) Gaines, G. L. Insoluble Monolayers at Liqid Gas Interface; Wiley: New York, 1966.

(33) Smith, T. Adv. Colloid Interface Sci. 1972, 3, 161.

(34) Kaganer, V. M.; Möhwald, H.; Dutta, P. Rev. Mod. Phys. 1999, $71,779$.

(35) (a) Deutsch, M.; Ocko, B. M. In Encyclopedia of Applied Physics; Trigg, G. L. Ed.; VCH: New York, 1998; Vol. 23, p 479. (b) Als-Nielsen, J.; McMorrow, D. Elements of Modern X-Ray Physics; Wiley: New York, 2001.
(36) Ocko, B. M.; Wu, X. Z.; Sirota, E. B.; Sinha, S. K.; Gang, O.; Deutsch, M. Phys. Rev. E 1997, 55, 3164.

(37) Braslau, A.; Pershan, P. S.; Swislow, G.; Ocko, B. M.; Als-Nielsen, J. Phys. Rev. A 1988, 38, 2457.

(38) (a) Vineyard, G. H. Phys. Rev. B 1982, 26, 4146. (b) Dietrich, S.; Haase, W. Phys. Rep. 1995, $260,1$.

(39) Magnussen, O. M.; Ocko, B. M.; Regan, M. J.; Penanen, A.; Deutsch, M.; Pershan, P. S. Phys. Rev. Lett. 1995, 74, 4444.

(40) (a) Flack, H. D. Philos. Trans. R. Soc. London, Ser. A 1970, 266, 561. (b) Reynolds, P. A. Acta Crystallogr., Sect. A 1975, 31, 80. 